\author{
L. P. Bron · D. Soldati • M.-L. Monod • C. Mégevand \\ E. Brossard · P. Monnier • P. Pasche
}

\title{
Horizontal partial laryngectomy for supraglottic squamous cell carcinoma
}

Received: 6 November 2003 / Accepted: 7 June 2004 / Published online: 14 August 2004

(C) Springer-Verlag 2004

\begin{abstract}
Between 1981-1999, 75 patients treated for supraglottic SCC with horizontal supraglottic laryngectomy (HSL) at the Otolaryngology Head and Neck Surgery Department of Lausanne University Hospital were retrospectively studied. There were 16 patients with T1, 46 with T2 and 13 with T3 tumors. Among these, 16 patients $(21 \%)$ had clinical neck disease corresponding to stage I, II, III and IV in 12, 39, 18 and 6 patients, respectively. All patients had HSL. Most patients had either elective or therapeutic bilateral level II-IV selective neck dissection. Six patients $(8 \%)$ with advanced neck disease had ipsilateral radical and controlateral elective II-IV selective neck dissections. Adjuvant radiotherapy was given to 25 patients $(30 \%)$ for either positive surgical margins $(n=8)$, pathological nodal status $(n=14)$ or both $(n=3)$. Median followup was 48 months (range, 24-199). Five-year disease-specific survival and locoregional and local control were 92, 90 and $92.5 \%$, respectively. Among five patients who were diagnosed with local recurrence, one had a total laryngectomy $(1.4 \%)$; the others were treated by endoscopic laser surgery. Two patients had both a local and regional recurrence. They were salvaged with combined surgery and radiotherapy, but eventually died of their disease. Cartilage infiltration seems to influence both local control $(P=0.03)$ and disease-specific survival $(P=0.06)$. There was a trend for worse survival with pathological node involvement $(P=0.15)$ and extralaryngeal extension of the cancer $(P=0.1)$. All patients except one recovered a close to normal function after the treatment. Aspiration was
\end{abstract}

This paper was presented at the 2nd World Congress of the International Federation of Head and Neck Oncologic Societies, Rio de Janeiro, Brazil, 29 November-2 December 2002.

L. P. Bron (困) · D. Soldati · M.-L. Monod · C. Mégevand

E. Brossard · P. Monnier · P. Pasche

Department of Otorhinolaryngology and Head and Neck Surgery, Centre Hospitalier Universitaire Vaudois, CHUV BH-12/709,

1011 Lausanne, Switzerland

Tel.: +41-21-3142682,

e-mail: luc.bron@chuv.hospvd.ch present in 16 patients (26\%) in the early postoperative period. A median of 16 days (7-9) was necessary to recover a close to normal diet. Decannulation took a median of 17 days (8-93). Seven patients kept a tracheotomy tube for up to 3 months because of persistent aspiration. There was no permanent tracheostomy or total laryngectomy for functional purposes. Horizontal supraglottic laryngectomy remains an adequate therapeutic alternative for supraglottic squamous cell carcinoma, offering an excellent oncological outcome. The postoperative functional morbidity is substantial, indicating the need for careful patient selection, but good laryngeal function recovery is the rule. The surgical alternative is endoscopic laser surgery, which may offer comparable oncological results with less functional morbidity. Nevertheless, these two different techniques need to be compared prospectively.

Keywords Supraglottic squamous cell carcinoma • Horizontal partial laryngectomy $\cdot$ Functional laryngeal surgery

\section{Introduction}

There is no real consensus on the best treatment option for supraglottic squamous cell carcinoma. Single modalities using either surgery or radiotherapy alone or combined sequential therapy are different therapeutic options available $[1,2]$. In 1947, Alonso [3] described horizontal partial laryngectomy (HPL) as a surgical treatment for supraglottic squamous cell carcinoma. This technique took several decades to gain widespread recognition, but, in the past 20 years, it has progressively replaced total laryngectomy for selected cancers. Radiation therapy is a recognized alternative treatment allowing preservation of laryngeal function. Provided patients are carefully selected, both treatments appear to offer comparable oncological and functional outcomes $[4,5,6,7]$.

In our department, functional laryngeal surgery has been the main line of treatment for selected glottic and supraglottic SCC for 20 years $[8,9]$. We have usually kept en- 
doscopic treatment as an alternative treatment to radiotherapy for early glottic cancers [10]. HPL, as described by Alonso [3], was mostly used for healthy patients presenting with stage II and above SCCs of the supraglottis. Advanced chronic respiratory disease was considered a contraindication. Thus, radiotherapy was kept as an adjuvant treatment or as a primary treatment modality for those patients medically unfit for functional laryngeal surgery still trying to avoid the morbidity of total laryngectomy.

Progressively introduced in the last 10 years, transoral $\mathrm{CO}_{2}$ laser excision combined with surgery and/or radiotherapy to the neck seems to offer a valuable surgical alternative for the treatment of supraglottic primaries, with a more rapid recovery of laryngeal function $[11,12,13]$. We have recently introduced this technique in our department and are currently studying our results prospectively. During the past 18 years, our standard approach has been an open horizontal partial laryngectomy. This retrospective study of 75 patients analyzes the postoperative course, mortality and morbidity of this surgical approach to supraglottic cancer.

\section{Subjects and methods}

During the period of 1981 to 1999,75 supraglottic partial laryngectomies were performed in the department of Otolaryngology and Head and Neck Surgery of Lausanne University Hospital. All clinical and pathological data were reviewed retrospectively. Among the 75 patients, there were 70 male and 5 female patients with a median age of 58 years (range, 32-75). The staging according to the 1983 American Joint Committee on Cancer Staging (AJCCS) is listed in Table 1.

Follow-up data were collected through bimonthly visits in our department for the first 2 years, extended to twice a year for the following years. Median follow-up was 48 months (range, 24199). All patients presented with supraglottic squamous cell carcinoma. The tumor was confined to the laryngeal surface of the epiglottis extending into the larynx and the valleculae or tongue base in $68(91 \%)$ and $7(9 \%)$ patients, respectively. Patients with lateral extension to the pharynx were excluded. One patient had been treated previously with radiotherapy for a SCC of the epiglottis.

All patients had a pretherapeutic panendoscopy, including rigid broncho-esophagoscopy and direct laryngoscopy, to determine the extent of the tumor accurately and define the indication for surgery. Surgical treatment included HPL, along with bilateral neck dissection. The pharynx was systematically open at the tongue base to locate the anterior commissure and resect the thyroid cartilage precisely above the glottis, along with the entire epiglottis and the preepiglottic space. A pexy was then performed between the tongue base and the lower third of the thyroid cartilage. Our indication for this surgery was cancer of the supraglottis without ex-

Table 1 Distribution by clinical $\mathrm{T}$ and $\mathrm{N}$ stage

\begin{tabular}{lrrrr}
\hline & T1 & T2 & T3 & \\
\hline N0 & 12 & 36 & 11 & 59 \\
N1 & 0 & 3 & 1 & 4 \\
N2 & 1 & 5 & 0 & 6 \\
N3 & 3 & 2 & 1 & 6 \\
Total & 16 & 46 & 13 & 75 \\
\hline
\end{tabular}

tension into the vocal cords and minimal extension into the tongue base. The extent of neck surgery was dependent on the clinical status of the neck nodes, but all patients had at least a bilateral selective neck dissection, preserving the submandibular triangle, internal jugular vein, sternocleidomastoid muscle and spinal accessory nerve. Indication for adjuvant radiotherapy was as follows: positive or insufficient surgical margins and multiple neck nodes or extracapsular spread. If needed, radiotherapy courses were scheduled within 6 weeks postoperatively and delivered weekly over a period of 6 to 10 weeks to reach a total dose of $45-60$ Gy. No patient had clinically positive neck disease treated with radiotherapy alone.

Statistical analysis was performed with JMP Statistics Made Visual software (SAS Institute Inc., Trunbull, Conn.). Means were compared by Student's $t$-test. Proportions were compared using a chi-square test for values greater than five and the Fisher's exact test for those less than or equal to five. Kaplan-Meier product-limit estimates were used to evaluate overall survival, cause-specific survival and local control in both groups. Univariate analysis of correlation of the following independent factors - age, tumor extension and pre- versus postoperative nodal disease - to local control and cause-specific survival was performed using the log-rank test. Multivariate analyses were done using the Cox stepwise regression analysis to determine the independent contribution of each prognostic factor.

\section{Results}

There were $16(21 \%)$ therapeutic and $59(79 \%)$ elective neck dissections. There were 69 bilateral selective level II-IV neck dissections, preserving the internal jugular vein, the spinal accessory nerve, the sternocleidomastoid muscle and the submandibular triangle. Six patients $(8 \%)$ with clinically advanced disease (N3) had an ipsilateral radical neck dissection and a contralateral, selective, level II-IV dissection. The submandibular triangle was dissected on the ipsilateral side in two patients who initially presented with an oral second primary, which was treated surgically, at the same time.

Histopathology showed close or involved surgical margins in 16 patients (21\%). Overall, 26 patients had pathologically positive lymph nodes (35\%); of these, ten cases $(15 \%)$ were occult nodal disease. Pathological staging is listed in Table 2. Adjuvant radiotherapy was indicated in 25 patients, by nodal status in 14 cases, positive surgical margins in 8 and both in 3 .

Minor postoperative complications such as hematomas and neck infections affected four and two patients, respectively. Two patients presented a dehiscent pexy in the immediate postoperative period, which was diagnosed and immediately treated surgically without long-term consequences for the patients. Other medical complications included one pulmonary embolism, one deep venous throm-

Table 2 Distribution by pathological $\mathrm{T}$ and $\mathrm{N}$ stage

\begin{tabular}{lrrrrr}
\hline & pT1 & pT2 & pT3 & pT4 & \\
\hline pN0 & 12 & 28 & 7 & 2 & 49 \\
pN1 & 1 & 6 & 4 & 0 & 11 \\
pN2 & 0 & 8 & 2 & 0 & 10 \\
pN3 & 3 & 1 & 0 & 1 & 5 \\
Total & 16 & 43 & 13 & 3 & 75 \\
\hline
\end{tabular}


Table 3 Univariate analysis in 75 patients for local control and disease-free survival

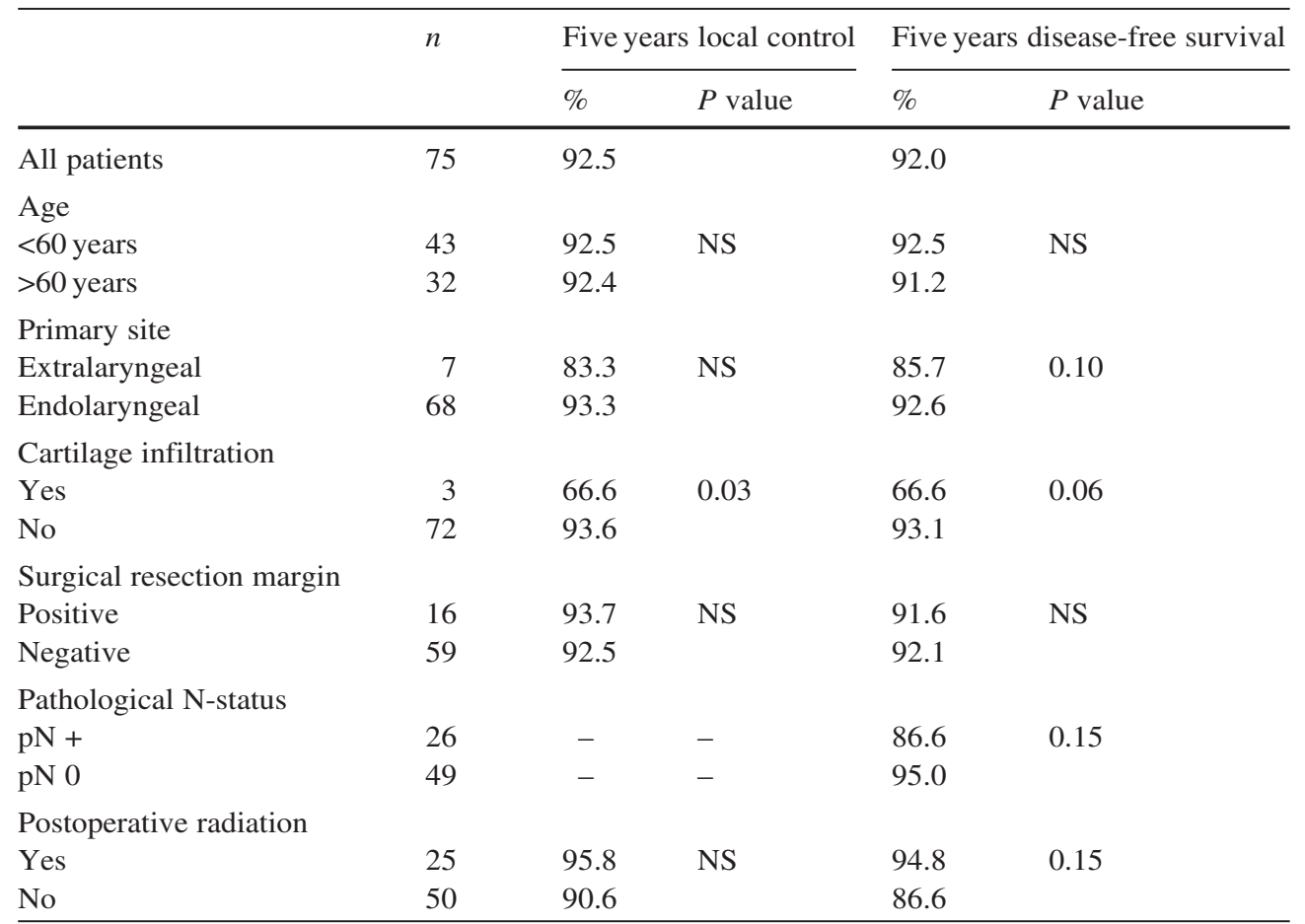

bosis and two pneumonias. They could all be managed during hospitalization. No patient died during the postoperative period.

The overall 5-year local and locoregional control rate was 92.5 and $90 \%$, respectively. Local recurrence only was the cause of failure in three patients. Two of them were salvaged surgically, retaining their larynx. One had a total laryngectomy for a deep recurrence in the tongue base. Another two patients had both a local and regional failure. They were treated with combined surgery and radiation therapy. One patient was salvaged, but the second died of his disease. Neck recurrence alone occurred in three patients. Salvage treatment was surgical and successful in one of them. Globally, five of eight locoregional recurrences were salvaged successfully. The ultimate 5-year locoregional control was then $95 \%$ with a $98.5 \%$ laryngeal preservation rate. With $100 \%$-year local control for pT1, $91 \%$ for pT2, 92 and $66 \%$ for pT3 and pT4, respectively, pathological T-stage did not appear to influence significantly local control for pT1-pT3 tumors. Probably due to the small number of recurrences encountered, infiltration of the larynx skeleton (pT4 vs. pT1-3) was the only variable showing an impact on local control $(P<0.03)$ (Table 3$)$.

Distant spread of disease was the cause of failure in six patients. Distant metastasis involved the liver, lung and bone. The overall 5-year incidence of distant metastasis was $6.9 \%$.

On initial panendoscopy, four patients $(5 \%)$ were diagnosed with early stage synchronous second primary cancers in the oral cavity, the larynx and the lung. During regular follow-up, 17 (23\%) metachronous cancers were diagnosed. They involved the upper aerodigestive tract, lung and esophagus in nine, seven and one patients, respectively. The median time of detection of these second
Table 4 Causes of death

\begin{tabular}{lcr}
\hline Cause of death & $\begin{array}{l}\text { No. of } \\
\text { patients }\end{array}$ & $\%$ \\
\hline TNM related death & & \\
Local recurrence & 1 & 2 \\
Neck recurrence & 2 & 5 \\
Metastasis & 4 & 12 \\
Second primary tumor & 9 & 3 \\
Other cancer & 2 & 24 \\
Intercurrent disease & 18 & \\
\hline
\end{tabular}

primaries was 71 months (range 4-200). Treatment was surgical in all cases, except for the esophageal second primary, which was diagnosed at a very early stage and was successfully treated with endoscopic photodynamic therapy. Of those 17 patients, 9 died from their second cancer.

The 5-year overall and cause-specific actuarial survival rates were 75 and $92 \%$, respectively. Causes of death are summarized in Table 4. Survival appeared not to be influenced by the size of the primary. Disease-specific survival by pathological T-stage was 93, 91, 100 and $66 \%$ for pT1, pT2, pT3 and pT4, respectively. Extension of the cancer into the cartilaginous framework of the larynx, however, seems to have an influence, but did not reach statistical significance. There was also a trend for worse survival when the primary cancer extends into the oropharynx and when neck nodes are involved. Because of the relatively small number of cases, none of these variables reached statistical significance (Table 3 ).

Postoperatively, the median time for decannulation was 17 days (range 8-93). For 16 patients (21\%), aspiration delayed closure of the tracheotomy. Of those, seven 
patients $(9 \%)$ were discharged with a tracheotomy and a nasogastric feeding tube. All of them were eventually decannulated and able to swallow in a maximum period of 3 months, providing intensive swallowing rehabilitation. One patient became aphagic after radiotherapy because of cricopharyngeal muscle stenosis. He then needed insertion of a percutaneous gastrostomy and has not been able to eat since, even after multiple endoscopic dilatations. There was no total laryngectomy for failure to recover function.

\section{Discussion}

Optimal management of supraglottic cancers aiming at the best possible cure and functional conservation has been a matter of debate between surgeons and radiation oncologists for many years. We have reviewed a series of 75 patients with supraglottic SCC treated primarily with HPL.

Cancers of the supraglottis are efficiently treated with functional surgery, which achieves a very good local control of the disease in early lesions. But, more advanced primaries are less likely to be treated by surgery alone, notably when the tumor extends into the base of the tongue, laryngeal cartilages or paralaryngeal spaces [4, 5, 14]. For stage I and II diseases, both Bocca et al. [4] and DeSanto [5] report between $85-100 \%$ local control at 3 years after treatment with HPL in series of 537 and 70 patients, respectively. Advanced stage of disease is usually accompanied with a drop in local control, in particular for T4 cancers $[4,15]$. Most of the recurrences present in the tongue base, but salvage treatment is efficient, even if it often requires a total laryngectomy. Adjuvant radiotherapy improves both local control and survival in cases with advanced neck disease or positive surgical margin and is therefore considered standard in these settings $[14,16]$.

With primary radiotherapy more so than surgery, recurrence rates are dependent on the primary tumor volume and the degree of pre-epiglottic space infiltration, as seen on CT examination [17]. Local control varies greatly with stage between 100 to $27 \%$ from T1 to T4 cancers, respectively $[7,18]$. In case of recurrence, surgical salvage is efficient, but requires a total laryngectomy in up to $30 \%$ of cases, even for patients initially eligible for a functional procedure $[7,18,19]$. Failure in the neck is a significant problem in supraglottic cancer and impacts on patient survival $[2,6,15,16]$. Whereas positive neck nodes require combined surgery and radiotherapy $[7,16]$, the management of the clinically negative neck at present is still controversial.

The percentage of occult nodal disease is around $30 \%$ $[5,6,20]$. Unilateral treatment, based on the initial localization of the primary, is insufficient and carries a high risk of failure in the contralateral neck $[5,6,15]$. Bilateral irradiation appears effective in eradicating occult metastasis in both sides of the neck, but carries significantly more long-term morbidity than elective surgical treatment [5, 6]. It therefore has been our attitude to perform systemat- ically bilateral anterior (level II-IV) neck dissection as both a therapeutic and diagnostic measure and to use combined therapy, with adjuvant radiotherapy, when necessary. Our findings of 35\% pathological neck disease and our extremely low rate of regional recurrence $(8 \%)$ support our practice. Despite the relatively low percentage of occult nodal disease $(15 \%)$ in our series, we preferred bilateral selective neck dissection, preserving the submandibular triangle, internal jugular vein, sternocleidomastoid muscle and spinal accessory as it carries hardly no morbidity compared to a bilateral prophylactic irradiation.

Using primary surgery and adjuvant radiotherapy, our global 5-year local control rate was $92.5 \%$. With 100,91 , 92 and $66 \%$ for T1, T2, T3 and T4, respectively, our results compare favorably with previously published data $[4,5,14,15]$. Among eight patients who recurred, three failed at the primary site only. They could all be salvaged, with one patient losing his larynx. The other five patients failed in the neck. Combined surgery and radiotherapy salvaged two patients, but three could not be controlled and eventually died of their disease. Our 5-year overall loco-regional control was then $95 \%$ with a 98.5 laryngeal conservation rate.

Despite our good $92 \%$ disease-free survival rate at 5 years, the overall 5-year survival was $75 \%$, mainly because of second primary cancers. Mostly found in the lung, they are diagnosed on routine follow-up radiological examination and often confirmed endoscopically. The high incidence of metachronous cancers is a recognized problem of head and neck cancers, especially in survivors of laryngeal cancers $[9,21]$. Despite systematic pretherapeutic broncho-esophagoscopy, regular postoperative followup and broncho-esophageal cytological studies with control panendosocopies at 2 years, patients appeared to present with aggressive second primaries in the lung, which are often fatal [22]. In addition, with such a high locoregional control rate, distant metastases are beginning to have a significant impact on survival, a phenomenon that we have previously noticed in glottic cancers [9]. Some authors advocate the use of neoadjuvant chemotherapy before surgery for laryngeal cancers. Thus, they intend to control some of the early tumoral dissemination and effectively achieve better survival [23].

Our functional results were satisfactory. Normal laryngeal function, with removal of the tracheostomy tube and a close to normal diet, was reached in $80 \%$ of the patients within a month. Recovery of a good swallowing function was delayed in 16 patients. However, they were all decannulated and able to eat in the first 3 months after the surgery. No completion laryngectomy for intractable aspiration was needed. With $98.5 \%$, our overall laryngeal preservation rate compares favorably with previously published data reporting 98 to $93 \%$ laryngeal preservation rates after surgery $[14,24]$ and $84 \%$ after radiotherapy [18]. Recovering a close to normal swallowing function after HPL is often a major problem, and an increased incidence of life-long gastrostomy has been reported [25, 26]. In our series, only one patient, who presented with cricopharyngeal muscle stenosis after adjuvant radiother- 
apy, required a percutaneous gastrostomy and never recovered normal swallowing, despite multiple attempts at endoscopic dilatation. However, open surgery of the larynx remains a serious undertaking for some patients whose general health condition, notably respiratory, may not allow the transient postoperative loss of laryngeal function.

\section{Conclusion}

With careful selection of the patients, HPL, along with adjuvant radiotherapy, is a suitable treatment option for supraglottic SCC. Postoperative recovery of laryngeal function is the rule, and long-term sequelae are infrequent, with $60 \%$ of the patients avoiding adjuvant treatment. For the initial early stage of the disease (stages I-II), radiotherapy seems to offer a similar outcome with less immediate functional morbidity. However, in patients initially suitable for functional surgery, salvage of local failure most often requires a total laryngectomy. Based on these results, our first line of treatment for surpaglottic cancers remains surgical.

Transoral $\mathrm{CO}_{2}$ laser resection of these cancers controls early stages of the disease with efficiency comparable to open surgery and carries less laryngeal morbidity. This fairly new approach has brought promising results and offers an excellent alternative treatment for selected patients. Following the publication of numerous convincing reports, we have recently introduced transoral $\mathrm{CO}_{2}$ laser resections in our department and are prospectively collecting data to compare endoscopic with open transcervical horizontal partial laryngectomies

\section{References}

1. DeSanto LW (1985) Cancer of the supraglottic larynx: a review of 260 patients. Otolaryngol Head Neck Surg 93:705-711

2. Spaulding CA, Hahn SS, Constable WC (1987) The effectiveness of treatment of lymph nodes in cancers of the pyriform sinus and subglottis. Int J Radiat Oncol Biol Phys 13:963-968

3. Alonso JM (1947) Conservative surgery of cancers of the larynx. Trans Am Acad Ophtalmol Otolaryngol 51:633-642

4. Bocca E (1991) Surgical management of supraglottic cancer and its lymph nodes metastases in a conservative perspective. Ann Otol Rhinol Laryngol 100:261-267

5. DeSanto LW (1990) Early supraglottic cancer. Ann Otol Rhinol Laryngol 99:593-597

6. Levendag P, Vikram B (1987) The problem of neck relapse in early stage supraglottic cancer - results of different treatment modalities for the clinically negative neck. Int J Radiat Oncol Biol Phys 13:1621-1624

7. Mendenhall WM, Pearsons JT, Mancuso AA (1996) Radiotherapy for squamous cell carcinoma of the larynx: an alternative to surgery. Head Neck 18:24-35

8. Bron L, Brossard E, Monnier P, Pasche P (2000) Supracricoid partial laryngectomy with cricohyoidoepiglottopexy and cricohyoidopexy for glottic and supraglottic carcinomas. Laryngoscope 110:627-634
9. Bron LP, Pasche P, Monnier P, Schweizer V (2002) Functional analysis after supracricoid partial laryngectomy with CHEP or CHP. Laryngoscope 112:1289-1293

10. Bron LP, Soldati D, Zouhair A, Ozsahin M, Brossard E, Monnier P, Pasche P (2001) Treatment of early stage squamous cell carcinoma of the glottic larynx: endoscopic surgery or cricohyoidoepiglottopexy versus radiotherapy. Head Neck 23: 823-829

11. Ambrosch P, Kron M, Steiner W (1998) Carbon dioxide laser microsurgery for early supraglottic carcinoma. Ann Otol Rhinol Laryngol 107:680-688

12. Iro H, Waldfahrer F, Altendorf-Hofmann A, Weidenbecher M, Sauer R, Steiner W (1998) Transoral laser surgery for supraglottic cancer. Arch Otolaryngol Head Neck Surg 124:12451250

13. Rudert HH, Werner JA, Höft S (1999) Transoral carbon dioxide laser resection of supraglottic carcinoma. Ann Otol Rhinol Laryngol 108:819-827

14. Lee NK, Goepfert H, Wendt CD (1990) Supraglottic laryngectomy for intermediate-stage cancer: U.T. M.D. Anderson cancer center experience with combined therapy. Laryngoscope 100:831-836

15. Lutz CK, Johnston JT, Wagner RL, Myers EN (1990) Supraglottic carcinoma: patterns of recurrence. Ann Otol Rhinol Laryngol 99:12-17

16. Nicolai P, Redalli LO, Tomenzoli D, Barezzani MG, Bertoni F, Bignardi M, Antonelli AR (1997) Prognostic determinants in supraglottic carcinoma: univariate and Cox regression analysis. Head Neck:323-334

17. Freeman DE, Mancuso AA, Parsons JT, Mendenhall WM, Million RR (1990) Irradiation alone for supraglottic larynx carcinoma: can CT findings predict treatment results. Int J Radiat Oncol Biol Phys 19:485-490

18. Spaulding CA, Krochak RJ, Hahn SS, Constable WC (1986) Radiotherapeutic management of cancer of the supraglottis. Cancer 57:1292-1298

19. Mendenhall WM, Parsons JT, Stringer SP, Cassissi NJ (1997) Radiotherapy for carcinoma of the supraglottis. Otolaryngol Clinics North Am 30:145-161

20. Spaulding CA, Constable WC, Levine PA, Cantrell RW (1989) Partial laryngectomy and radiotherapy for supraglottic cancer: a conservative approach. Ann Otol Rhinol Laryngol 98:125-129

21. Grosjean P, Pasche P, Monnier P (2000) Die Frühdiagnose von Karzinomen im Kopf- und Halsbereich. Interventionelle und diagnostische Bronchologie. Herausgegeben K. Wassermann. München-Deisenhofen. Dustri-Verlag

22. Licciardello JT, Spitz MR, Hong MPH, Hong WK (1989) Multiple primary cancer in patients with cancer of the head and neck: second cancer of the head and neck, oesophagus and lung. Int J Radiat Oncol Biol Phys 17:467-476

23. Laccourreye O, Muscatello L, Laccourreye L, Naudo P, Brasnu D, Weinstein G (1997) Supraacricoid partial laryngectomy with cricohyoidoepiglottopexy for "early" glottic carcinoma classified as T1-T2N0 invading the anterior commissure. Am J Otolaryngol 18:385-390

24. Bocca E, Pignataro O, Oldini C (1983) Supraglottic laryngectomy: 30 years of experience. Ann Otol Rhinol Laryngol 92: 14-18

25. Spriano G, Antognoni P, Sanguinetti G, Sormani M, Richetti A, Ameli F, Piantanida R, Luraghi R, Magli A, Corvo R, Tordiglione M, Vitale V (2000) Laryngeal long-term morbidity after supraglottic laryngectomy and postoperative radiation therapy. Am J Otolaryngol 21:14-21

26. Steiniger JR, Parnes SM, Gardner GM (1997) Morbidity of combined therapy for the treatment of supraglottic carcinoma: supraglottic laryngectomy and radiotherapy. Ann Otol Rhinol Laryngol 106:151-158 